\title{
Estigma social hacia la enfermedad mental: factores relacionados y propiedades psicométricas del Cuestionario de Atribuciones-revisado*
}

\section{Social Stigma towards Mental Illness: Related Factors and Psychometric Properties of the Revised-Attribution Questionnaire}

\author{
Ana Rodríguez-Meirinhos a \\ Universidad Loyola Andalucía, España \\ ORCID: https://orcid.org/0000-0002-1073-8151 \\ Lucía Antolín-Suárez \\ Universidad de Sevilla, España \\ ORCID: https://orcid.org/0000-0001-7333-7569
}

a Autor de correspondencia. Correo electrónico: arodriguezm@uloyola.es

Para citar este artículo: Rodríguez-Meirinhos, A., \& Antolín-Suárez, L. (2020). Estigma social hacia la enfermedad mental: factores relacionados y propiedades psicométricas del Cuestionario de Atribuciones-revisado. Universitas Psychologica, 19. https://doi.org/10.11144/Javeriana.upsy19. esem

\section{RESUMEN}

Este estudio persiguió analizar la estructura interna de la versión española del Cuestionario de Atribuciones-revisado ( $\mathrm{r}$-AQ-E) para evaluar el estigma social hacia la enfermedad mental en la adolescencia, así como investigar su relación con la edad, la familiaridad y el conocimiento sobre la enfermedad mental. La muestra estuvo constituida por 861 adolescentes de entre 12 y 16 años $(M=13.91 ; D E=1.22)$. Los análisis factoriales exploratorios y confirmatorios no replicaron la estructura original del $r$-AQ-E, y revelaron una estructura unidimensional con cinco ítems y adecuada consistencia interna. Los resultados evidenciaron que los chicos mostraban más estigma que las chicas. En ambos casos, los niveles de estigma eran similares entre los 12 y 15 años, siendo inferiores a los 16 años. El estigma social se relacionaba con la familiaridad y el conocimiento sobre las características, la prevalencia y las causas de las enfermedades mentales. Estos resultados enfatizan la necesidad de implementar intervenciones para reducir el estigma mediante la mejora del conocimiento sobre la enfermedad mental y las oportunidades de contacto directo.

Palabras clave

estigma; enfermedad mental; r-AQ; validez; conocimiento; familiaridad.

\begin{abstract}
The aim of this study was to analyze the internal structure of the revisedAttribution Questionnaire Spanish version (r-AQ-E) to assess social stigma towards mental illness in adolescents, as well as, to investigate its relationship with age, familiarity, and knowledge about mental illness. The sample was composed by 861 adolescents, aged between 12 and 16 yearsold $(M=13.91 ; S D=1.22)$. The Exploratory and Confirmatory Factor Analyses did not replicate the original structure of the $\mathrm{r}-\mathrm{AQ}-\mathrm{E}$, but fitted a unidimensional structure with 5 items and adequate internal consistence.
\end{abstract}


Results evidenced that boys showed more stigma than girls. In both groups, levels of stigma were not significantly different between the age of 12 and 15, being lower at 16. Social stigma was related with familiarity and knowledge about characteristics, prevalence, and causes of mental illness. These findings highlight the need to implement interventions to reduce stigma by improving the knowledge about mental illness and offering opportunities of direct contact.

Keywords

stigma; mental illness; r-AQ; validity; adolescent attitudes.

El estigma social hacia la enfermedad mental es un fenómeno universal, presente desde la infancia, que genera rechazo y discriminación hacia las personas que sufren problemas de salud mental (Wahl, 2002).

En los últimos años, ha sido reconocido por los principales organismos internacionales como una preocupación social debido al incremento alarmante y la alta prevalencia de enfermedades mentales tanto en población adulta como adolescente (Vigo, Thornicroft, \& Atun, 2016). Esto hace que cada vez sean más las personas que tienen que lidiar con un doble problema: la enfermedad en sí y el rechazo asociado. Las consecuencias del estigma social pueden llegar a ser tan dañinas como los propios síntomas de la enfermedad (Petersen, Soegaard, Lodahl, Vinther, \& Wind, 2014), generando dificultades tales como aislamiento social, soledad, obstaculización del acceso al mercado laboral, limitación de la autonomía por el paternalismo y la infantilización e incluso sentimientos de vergüenza, inferioridad y autorrechazo que acaban por instalarse en las personas que las sufren (Hinshaw, 2006; Quinn, Williams, \& Weisz, 2015; Seacat, 2014). En el caso de los chicos y chicas adolescentes, se añaden otras dificultades que pueden interferir con su desarrollo psicosocial como son el rechazo o las burlas de los compañeros de clase y la dificultad para hacer nuevos amigos o tener relaciones de pareja (Martínez \& Hinshaw, 2016; O'Driscoll, Heary, Hennessy, \& McKeague, 2015). Además, el estigma supone una de las principales barreras para la detección precoz y la recuperación, ya que la vergüenza y el miedo al rechazo pueden llevar a ocultar los síntomas, negarse a pedir ayuda o seguir los tratamientos (Clement et al., 2015). Las repercusiones de no detectar y tratar un problema de salud mental a tiempo pueden ser muy severas, afectando a la salud general y acabar derivando en una condición discapacitante (Wainberg et al., 2017). Por todo ello, la lucha contra el estigma, especialmente en la adolescencia como etapa en la que los problemas de salud mental suelen aparecer (Organización Mundial de la Salud, 2018), constituye una prioridad.

Desde que en la década de los 80 comenzó a surgir el interés por el estudio de las actitudes hacia la enfermedad mental, se han iniciado reformas para mejorar la integración de las personas que las sufren, así como campañas para superar los mitos (National Academies of Sciences, Engineering, and Medicine, 2017). Sin embargo, aunque ha aumentado la visibilidad y la conciencia, el rechazo y la discriminación continúa siendo tan alarmante como hace décadas (Casados, 2017). Fruto de ello, en el ámbito académico, se ha observado un creciente interés por comprender qué factores se asocian al estigma y cómo pueden contribuir a su reducción. Aunque estos estudios se han desarrollado fundamentalmente con población adulta, sirven como punto de partida para comprender el fenómeno del estigma social hacia la enfermedad mental en la adolescencia (Mukolo, Heflinger, \& Wallston, 2010).

Una de las variables que ha recibido más atención es la familiaridad o contacto previo con la enfermedad mental. Las investigaciones apoyan que el contacto interpersonal con miembros del grupo estigmatizado permite descreer, a través de la propia experiencia, el crédito estereotipado dado, reduciendo el rechazo y generando más empatía y conductas prosociales (Pettigrew \& Tropp, 2008). También, las evidencias derivadas de campañas y programas de intervención sugieren que ofrecer oportunidades para interactuar con personas con problemas de salud mental representa una estrategia eficaz para reducir el estigma (Casados, 2017; Chisholm et al., 2016). No obstante, aunque los datos son bastante concluyentes 
cuando se trata de adultos, no está claro si estas conclusiones son extensibles a poblaciones más jóvenes porque, en algunos estudios desarrollados con adolescentes, se han observado relaciones en el sentido opuesto. Por ejemplo, Corrigan et al. (2005) encontraron que aquellos adolescentes que habían tenido más contacto previo con personas con enfermedad mental las consideraban más peligrosas.

Otro aspecto asociado con el estigma es el conocimiento acerca de qué son y cómo afectan las enfermedades mentales. Los estudios de opinión reflejan una imagen social de la enfermedad mental cargada de desconocimiento y creencias poco ajustadas a la realidad (Angermeyer \& Dietrich, 2006). Entre estas, destacan que las personas con enfermedad mental son peligrosas, dependientes, débiles, raras, solitarias, suponen una carga para la sociedad, no pueden trabajar de cara a la gente, etc. (Reavley \& Jorm, 2011). Angermeyer, Beck y Matschinger (2003) argumentan que tener un conocimiento más ajustado sobre qué son los problemas de salud mental y las dificultades que generan, se asocia con menos prejuicios y conductas de discriminación. No obstante, hasta la fecha, no se ha investigado de manera sistemática qué tipo concreto de conocimiento (conocimiento sobre causas, síntomas, consecuencias...) se relaciona con menos estigma.

Otras variables frecuentemente analizadas en población adulta, aunque sin evidencias concluyentes, han sido las sociodemográficas. Respecto al género, los resultados son bastante inconsistentes. Aunque algunos estudios encuentran que el estigma no depende del género (Schnittker, 2000), otros observan que los hombres estigmatizan más que las mujeres (e. g. Holman, 2015; Pascucci et al., 2017). En cuanto a la edad, Schomerus, Van der Auwera, Matschinger, Baumeister y Angermeyer (2015) sugieren que las actitudes mantenidas hacia las personas con problemas de salud mental tienden a deteriorarse a lo largo del ciclo vital. No obstante, no está claro si estas diferencias se deben a que realmente las personas estigmatizan más con la edad o si se trata de un efecto de los cohortes generacionales. En cualquier caso, aun asumiendo que el estigma se relacione positivamente con la edad, como los datos provienen de estudios desarrollados con adultos, no informan de las diferencias en los niveles de estigma a lo largo de la adolescencia.

En definitiva, el estigma social hacia la enfermedad mental constituye un problema de enorme repercusión social sobre el que aún queda mucho por entender. En este sentido, el Pacto Europeo para la Salud Mental y el Bienestar (European Comission, 2008) recoge la necesidad de identificar factores implicados en su desarrollo e incorporarlos a las propuestas de intervención. Pese a ello, la investigación en la adolescencia todavía es escasa. Probablemente, una de las razones que lo justifica tiene que ver con la escasez de instrumentos adaptados y validados para evaluar este fenómeno en esta población.

En el contexto anglosajón uno de los instrumentos más conocidos y ampliamente utilizados para evaluar el estigma social hacia la enfermedad mental en adolescentes es el Cuestionario de Atribuciones-revisado o revisedAttribution Questionnaire ([r-AQ]; Watson et al., 2004) que se compone de ocho ítems, cada uno de los cuales evalúa un componente del estigma social, dos relativos a estereotipos (peligrosidad y culpabilidad), tres relacionados con prejuicios (ausencia de pena, ira y miedo) y tres referentes a conductas discriminatorias (segregación, evitación y rechazo de ayuda). Este cuestionario constituye una versión revisada del Attribution-Questionnaire-27 ([AQ-27]; Corrigan, Markowitz, Watson, Rowan, \& Kubiak, 2003), diseñado para población adulta. Ambos instrumentos se fundamentan en el modelo teórico propuesto por la Teoría de la Atribución (Weiner, 1995). Este modelo plantea que el origen del estigma está en los estereotipos o estructuras de conocimiento (generalmente inapropiadas o inexactas) compartidas por la sociedad. Su aceptación genera prejuicios o reacciones emocionales negativas y en última instancia conductas de discriminación que sitúan a la persona con enfermedad mental en una posición de inferioridad (Corrigan \& Kosyluk, 2014). 
En la actualidad, la versión para adultos, AQ-27, ha sido validada con éxito en distintos idiomas incluido el español (Muñoz, Guillén, Pérez-Santos, \& Corrigan, 2015). En contraste, las evidencias de validez de la versión para adolescentes, $r-A Q$, son muy escasas. En el estudio original (Watson et al., 2004), los autores únicamente proporcionaron evidencias de la adecuada consistencia interna de la escala. En cuanto a su estructura factorial, asumieron que, con base en motivos teóricos, el r-AQ se compondría de las mismas dimensiones que el AQ-27, pero no se desarrollaron análisis factoriales para examinar empíricamente su estructura interna. Posteriormente, Pinto, Hickman, Logsdon y Burant (2012) analizaron la dimensionalidad del $r$-AQ y propusieron una versión modificada con una estructura unidimensional compuesta por cinco ítems. Por tanto, aunque el $r$-AQ es un instrumento teóricamente fundamentado que ofrece una medida breve del estigma social hacia la enfermedad mental en adolescentes, no existe acuerdo en cuanto a sus propiedades psicométricas, siendo especialmente limitadas e incluso controvertidas, las evidencias en cuanto a la estructura de la escala (Wei, McGrath, Hayden, \& Kutcher, 2018).

Esta investigación, por tanto, se centra en el estudio del estigma social hacia la enfermedad mental en la adolescencia. A tal fin, se formularon los siguientes objetivos y se enunciaron las siguientes hipótesis. En primer lugar, considerando la necesidad de contar con evidencias del comportamiento psicométrico de los instrumentos para evaluar el estigma social en adolescentes, este estudio persigue analizar la estructura interna de la versión española del r-AQ (Watson et al., 2004). En segundo lugar, analizar diferencias en los niveles de estigma social hacia la enfermedad mental en función de la edad de los chicos y chicas adolescentes, así como investigar la posible relación del conocimiento y la familiaridad con la enfermedad mental. Dada la falta de consenso en los antecedes de la investigación, no se formularon hipótesis sobre el papel de la edad, aunque se esperaba que las chicas mostraran niveles de estigma más bajos que los chicos. Asimismo, se planteó la hipótesis de que niveles más elevados de conocimiento sobre la enfermedad mental y un mayor grado de familiaridad se relacionarían con menores niveles de estigma.

\section{Método}

\section{Participantes}

La muestra estuvo constituida por 861 adolescentes (428 chicos, $49.7 \%$ ) de 12 a 16 años $(\mathrm{M}=13.91 ; \mathrm{DE}=1.22)$ que estudiaban Educación Secundaria Obligatoria (ESO) en cuatro centros públicos y dos concertados de Sevilla (España). La nacionalidad mayoritaria era la española $(95.7 \%)$ y el $32.5 \%$ procedía de centros educativos ubicados en zonas de nivel socioeconómico alto, el $40.8 \%$ de nivel medio y el $26.7 \%$ de nivel bajo.

\section{Instrumentos}

\section{Estigma social}

Para evaluar el estigma social se utilizó el instrumento Revised-Attribution Questionnaire (r-AQ; Watson et al., 2004) compuesto por ocho ítems en formato Likert de 1 a $7(1=$ totalmente en desacuerdo, 7 = totalmente de acuerdo). Cada ítem hace referencia a un componente del estigma social: peligrosidad, culpabilidad, ausencia de pena, ira, miedo, segregación, evitación y rechazo de ayuda. A los participantes se les pidió que leyeran una historia que describía a un nuevo compañero de clase que sufre problemas de salud mental, y respondieran reflexionando sobre qué pensarían, cómo se sentirían y cómo actuarían con este compañero. Información detallada sobre las propiedades psicométricas de este instrumento puede encontrarse en la sección de resultados. 
Familiaridad con la enfermedad mental

La familiaridad o contacto previo del adolescente con la enfermedad mental fue evaluada con el instrumento Level of Contact Report for Adolescents (Corrigan et al., 2005). Se compone de ocho ítems en los que se describen situaciones que varían desde el menor nivel de contacto con la enfermedad mental (e. g., nunca he visto a una persona con una enfermedad mental) al mayor (e. g., tengo una enfermedad mental). A los adolescentes se les da la consigna de marcar todas las opciones que reflejen su experiencia. El índice de familiaridad se obtiene asignando la puntuación correspondiente a la situación de mayor contacto indicada, de manera que el rango de puntuaciones varía de 0 a 7 (a mayor puntuación, mayor nivel de familiaridad).

\section{Conocimiento sobre la enfermedad mental}

Para medir qué saben los adolescentes sobre las enfermedades mentales, se diseñó un Inventario de Conocimientos sobre las Enfermedades Mentales compuesto por 24 ítems en formato Likert de 1 a $4(1=$ totalmente falso, $4=$ totalmente verdadero). Este inventario evalúa cinco áreas de conocimiento: prevalencia (e. g., "las mujeres sufren más problemas de salud mental que los hombres"), causas (e. g., "existen trastornos o enfermedades mentales que aparecen principalmente por problemas biológicos"), síntomas (e. g., "el síntoma principal de la esquizofrenia es el deseo de agredir a los demás"), tratamientos (e. g., "los tratamientos para la mayoría de las enfermedades o trastornos mentales no funcionan") y características de las personas afectadas (e. g., "un alumno o alumna con un trastorno o enfermedad mental difícilmente podrá tener éxito en sus estudios"). Para aportar evidencias de validez de contenido, los ítems fueron sometidos a un doble proceso de depuración: 1) juicio de expertos utilizando la orientación de Navas et al. (2001) para eliminar los ítems más problemáticos y 2) estudio piloto para comprobar la compresión de los ítems, su adecuada formulación y tiempo de respuesta. La consistencia interna calculada con alfa de Cronbach fue 0.56 para conocimiento sobre prevalencia, 0.64 para conocimiento sobre causas, 0.58 para conocimiento sobre síntomas, 0.61 para conocimiento sobre síntomas, 0.66 para conocimiento sobre tratamientos y 0.65 para conocimiento sobre características.

\section{Procedimiento}

Se accedió a la muestra a través de la colaboración con seis centros de ESO que fueron seleccionados mediante un muestreo por cuotas en función del nivel socioeconómico bajo, medio o alto del área donde se ubicaban de acuerdo a los datos de renta per cápita media y ocupación. De cada zona se seleccionaron dos centros educativos, y de cada centro se solicitó la participación de dos grupos de 1. a 4. ${ }^{\mathrm{o}}$ de la ESO. Los responsables de los centros fueron informados de los objetivos y el procedimiento del estudio, y a las familias se les solicitó su consentimiento informado. El alumnado cumplimentó colectivamente los cuestionarios de forma voluntaria y anónima, en presencia de un miembro del equipo investigador. Se garantizó la confidencialidad. Este proyecto contó con la aprobación del Comité de Ética de la institución de los investigadores.

\section{Análisis de datos}

Para la obtención de evidencias de validez cruzada, la estructura interna de la escala $r$ AQ fue examinada en dos fases consecutivas. En primer lugar, se realizó un análisis factorial exploratorio (AFE) con rotación geomín oblicua (que permite la correlación entre factores) con una mitad de la muestra seleccionada aleatoriamente $(N=431)$. Previamente, se desarrolló un análisis de validación de supuestos mediante el test de esfericidad de Barlett y el índice $\mathrm{KMO}$ de Kaiser-Meyer-Olkin para verificar la idoneidad de la matriz de datos. La determinación del número óptimo de factores por retener se basó en varios criterios (LloretSegura, Ferreres-Traver, Hernández-Baeza, \& 
Tomás-Marco, 2014): (1) Los resultados del análisis paralelo con 5000 simulaciones aleatorias que sugiere la extracción de los factores comunes que presentan autovalores mayores de los que se obtendrían por azar (Horn, 1965); (2) La comparación del ajuste global de modelos con un factor más y menos de lo sugerido en el análisis paralelo y (3) la interpretabilidad teórica de la solución analizada. Para la extracción de factores, también se consideraron otros criterios basados en el porcentaje de varianza explicada y un mínimo de tres ítems con significado congruente para representar un factor común. En cuanto a los ítems, se retuvieron aquellos con saturaciones superiores a 0.4 que además no presentaban cargas factoriales cruzadas $(<$ 0.32). Los ítems que no cumplieron este criterio fueron eliminados individualmente. El análisis se repitió hasta que se identificó la estructura factorial más parsimoniosa. En una segunda fase, se validó la estructura factorial obtenida en el AFE mediante un análisis factorial confirmatorio (AFC) con el resto de la muestra $(N=430)$. Finalmente, se evaluó la consistencia interna de la estructura resultante en la muestra total mediante el cálculo de los coeficientes alfa de Cronbach y alfa ordinal, además del coeficiente de confiabilidad compuesta.

Seguidamente, a partir de la solución factorial validada, se realizó un análisis univariado de la varianza (ANOVA) para analizar posibles diferencias en el estigma en función de la edad, el sexo y su efecto de interacción. Asimismo, se estimaron las correlaciones bivariadas entre el estigma social y varias variables de conocimiento y familiaridad con la enfermedad mental, y se desarrolló un análisis de regresión mediante modelización con ecuaciones estructurales para examinar la relación de estas variables con el estigma social.

Para los distintos análisis factoriales y la modelización con ecuaciones estructurales, se utilizó Mplus 7 (Muthén \& Muthén, 2012) con el estimador robusto de mínimos cuadrados ponderados (WLSMV) basado en matrices de correlaciones policóricas, recomendado en casos de no normalidad de los datos. El ajuste de los modelos fue evaluado mediante el estadístico chi cuadrado $\left(\chi^{2}\right)$ que sugiere que el modelo se ajusta a los datos cuando resulta no significativo, combinado con otros índices de ajuste relativos y absolutos menos sensibles al tamaño muestral: índice de ajuste comparativo (CFI), el índice de Tucker-Lewis (TLI), la raíz del error cuadrado de aproximación (RMSEA). Un buen ajuste está indicado por una combinación de valores de CFI y TLI superiores a 0.9 , junto con valores de RMSEA inferiores a 0.06 (Kline, 2011).

En relación con el tamaño de efecto, se tomaron como referencia los niveles convencionales de Cohen (1988) para el estadístico $r$ (pequeño $<0.3$, mediano $>0.3 \mathrm{y}$ $<0.5$, grande $>0.5$ ) y para la $\eta^{2}$ (pequeño $<$ 0.01 , mediano $>0.01$ y $<0.06$, grande $>0.14$ )

\section{Resultados}

Evidencias de validez de la escala r-AQ
basadas en la estructura interna:
Dimensionalidad y consistencia interna

El índice de adecuación muestral de $\mathrm{KMO}$ (0.68) y el test de esfericidad de Bartlett $\left(\chi^{2}(28)\right.$ $=323.22 ; p<0.001)$ confirmaron la factoriabilidad de la matriz de corrrelaciones. El análisis paralelo ${ }^{1}$ sugirió la extracción de un solo factor, ya que solo el primer autovalor fue superior al simulado (Figura 1).

\section{Figura 1}

Autovalores del análisis paralelo del r-AQ.

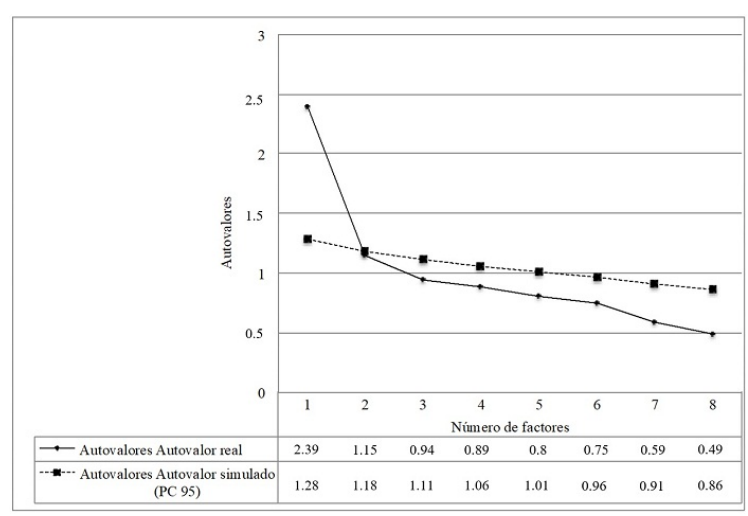


En términos de ajuste, las soluciones con uno, $\chi^{2}(20)=82.35, p<0.001$, CFI $=0.92$, TLI $=0.88 ;$ RMSEA $=0.08$ [CI $90 \%=0.06-0.1$ ], y con dos factores, $\chi^{2}(13)=39.36, p=0.002$, $\mathrm{CFI}=0.97$, TLI $=0.92$, $\mathrm{RMSEA}=0.07[\mathrm{CI}$ $90 \%=0.04-0.09$ ], mostraron un adecuado ajuste, siendo superior en el caso de dos factores. Sin embargo, al revisar la distribución de los ítems en la solución bifactorial se comprobó su escaso sentido a nivel teórico y metodológico. El primer factor, configurado por cuatro ítems, contribuía a explicar el $21.58 \%$ de la varianza y el segundo factor, explicaba el $12.56 \%$ de la varianza e incluía cuatro ítems, de los cuales dos presentaban pesos factoriales inferiores a 0.4 y un ítem mostraba pesos similares en ambos factores. Con objeto de seleccionar la estructura factorial más parsimoniosa y teóricamente interpretable, se limitó a uno el número de factores por extraer. Además, se eliminaron individualmente los ítems 1 "El nuevo alumno no es peligroso", 2 "Siento pena por el nuevo alumno" y 5 "El nuevo alumno no tiene la culpa por tener una enfermedad mental". La solución unifactorial obtenida mostraba un ajuste adecuado, $\chi^{2}(5)=$ $15.76, .=0.008, \mathrm{CFI}=0.98, \mathrm{TLI}=0.97$, RMSEA $=0.07$ [CI $90 \%=0.03-0.11$ ], $\mathrm{y}$ explicaba un 39\% de la varianza. Los estadísticos descriptivos y cargas factoriales para estos ítems pueden consultarse en la Tabla 1.

\section{Tabla 1}

Descriptivos y pesos factoriales de la solución final del AFE con cinco ítems

\begin{tabular}{lccc}
\hline \multicolumn{1}{c}{ Ítems } & $M$ & $D E$ & $\begin{array}{c}\text { Factor 1 } \\
(\lambda)\end{array}$ \\
\hline $\begin{array}{l}\text { 3. Manuel debería estar en un hospital para } \\
\text { enfermos mentales. }\end{array}$ & 2.5 & 1.74 & 0.54 \\
$\begin{array}{l}\text { 4. Yo intentaría mantenerme alejado del nuevo } \\
\text { alumno. }\end{array}$ & 2.52 & 1.68 & 0.83 \\
6. El nuevo alumno me haría sentirme enfadado. & 1.94 & 1.51 & 0.57 \\
7. Yo ayudaría al nuevo alumno. & 2.42 & 1.61 & 0.53 \\
8. Siento miedo del nuevo alumno. & 2.35 & 1.72 & 0.6 \\
\hline
\end{tabular}

Nota $M=$ Media, $D T=$ Desviación Tipo, $\lambda=$ Carga factorial de la solución rotada.

Los índices de bondad de ajuste del AFC sobre la solución resultante reflejaron la adecuación del modelo a los datos, $\chi^{2}(5)=19.29, p$ $=0.002$, CFI $=0.97$, TLI $=0.94$, RMSEA $=0.08[\mathrm{CI} 90 \%=0.04-0.11]$. Todos los coeficientes factoriales estandarizados resultaron estadísticamente significativos $(M=0.58)$. La consistencia interna calculada con alfa de Cronbach fue de $\mathbf{0 . 6 5}$, el coeficiente alfa ordinal fue de 0.75 y el coeficiente de confiabilidad compuesta fue de 0.72 .

Factores relacionados con el estigma social hacia la enfermedad mental

En la Tabla 2, se presentan las medias de estigma social para los chicos y las chicas en función de la edad (12, 13, 14, 15 y 16 años). Las chicas mostraban significativamente menos estigma que los chicos, $F(1,851)=6.15, p=0.013, \eta^{2}=$ 0.01 ( $M=2.40$ vs. $M=2.23$ chicos vs. chicas). También se encontraron diferencias en función de la edad, $F(4,851)=2.83, p=0.024, \eta^{2}=$ 0.01. En ambos sexos, los niveles de estigma no diferían entre los 12 y 15 años, siendo inferiores en el grupo de edad de 16 años. No obstante, en la prueba de comparaciones múltiples a posteriori de Tukey solo se detectó como significativa $(p=$ 0.029) la diferencia entre los grupos de 13 años $(M=2.43)$ y 16 años $(M=2.04)$. El efecto de interacción entre edad y género no resultó significativo lo que indica que la relación del estigma con la edad fue similar en chicos y chicas, $F(4,851)=0.473, p>0.05$.

\section{Tabla 2}

Medias y Desviaciones Típicas del estigma social en chicos y chicas de 12 a 16 años

\begin{tabular}{ccccccc}
\hline \multirow{2}{*}{ Age } & \multicolumn{3}{c}{ Hombres } & \multicolumn{2}{c}{ Mujeres } & \multicolumn{2}{c}{ Total } \\
\cline { 2 - 7 } & $M$ & $D E$ & $M$ & $D E$ & $M$ & $D E$ \\
\hline 12 & 2.48 & 1.08 & 2.22 & 1.01 & 2.34 & 1.05 \\
13 & 2.48 & 1.13 & 2.39 & 1.09 & 2.43 & 1.11 \\
14 & 2.24 & 1.13 & 2.19 & 0.86 & 2.21 & 0.98 \\
15 & 2.48 & 1.15 & 2.24 & 0.94 & 2.36 & 1.05 \\
16 & 2.19 & 0.99 & 1.86 & 0.83 & 2.04 & 0.93 \\
\hline Total & 2.4 & 1.11 & 2.23 & 0.97 \\
\hline \multicolumn{3}{c}{ Nota M $=$ Media, DT = Desviación Tipo } \\
\multicolumn{3}{c}{ En cuanto a la relación del r-AQ con }
\end{tabular}
otras variables, el estigma social correlacionaba negativamente con la familiaridad y el conocimiento sobre la prevalencia, las causas, los tratamientos y las características (Tabla 3). 
Tabla 3

Medias, desviaciones tipo y correlaciones entre variables de estudio.

\begin{tabular}{|c|c|c|c|c|c|c|c|}
\hline Variables & 1 & 2 & 3 & 4 & 5 & 6 & 7 \\
\hline $\begin{array}{l}\text { 1. } \mathrm{r}-\mathrm{AQ} \\
\text { 2. Familiaridad }\end{array}$ & $\begin{aligned} 1 \\
-0.3^{* * *}\end{aligned}$ & & & & & & \\
\hline 3. Conocimiento prevalencia & $-0.28^{* * *}$ & $0.14^{* * * *}$ & 1 & & & & \\
\hline 4. Conocimiento causas & $-0.21^{\cdots \cdots}$ & $0.09^{* *}$ & $0.17^{\cdots \cdots}$ & & & & \\
\hline 5. Conocimiento sintomas & -0.06 & $0.07^{*}$ & $0.09^{\circ}$ & $0.15^{* * *}$ & & & \\
\hline 6. Conocimiento tratamientos & $-0.17^{7 * \cdots}$ & $0.12^{2 *}$ & $0.23^{\cdots \cdots}$ & $0.13^{* * * *}$ & $0.18^{\circ * *}$ & 1 & \\
\hline 7. Conocimiento características & $-0.39^{9 * * x}$ & $0.2^{2+*}$ & $0.23^{* * *}$ & $0.22^{* * *}$ & $0.09^{* *}$ & $0.3^{* * *}$ & \\
\hline$M$ & 2.31 & 3.42 & 3.28 & 2.79 & 2.34 & 2.93 & 3.17 \\
\hline$D E$ & 1.05 & 1.4 & 1.46 & 0.48 & 0.42 & 0.51 & 0.48 \\
\hline
\end{tabular}

Además, mediante modelización con ecuaciones estructurales se evidenció que el modelo que relacionaba el estigma social con la familiaridad, y el conocimiento mostró un ajuste adecuado, $\chi^{2}(39)=85.80, p<0.001$, CFI $=$ 0.96 , TLI $=0.94$, RMSEA $=0.04[90 \% \mathrm{CI}=$ $0.03-0.05]$. El estigma social podría predecirse a partir de la familiaridad $(\beta=-0.22, p<0.001)$, el conocimiento sobre prevalencia $(\beta=-0.16, p$ $<0.001)$, sobre las causas $(\beta=-0.09, p=0.016)$ y sobre las características de las personas que las sufren $(\beta=-0.28, p<0.001)$. Puntuaciones más altas en estos predictores se relacionaban con menos estigma social. Además, el examen de los coeficientes de regresión estandarizados indicó que, de todas las variables anteriores, eran el conocimiento sobre las características seguido de la familiaridad las que realizaban una contribución más significativa. Asimismo, la proporción de varianza que estas variables explicaban sobre la puntuación global de estigma fue de $24 \%$.

\section{Discusión}

Los resultados de este estudio permitieron analizar las propiedades psicométricas de la versión española del cuestionario de Atribuciones-revisado, r-AQ (Watson et al., 2004) en una muestra de adolescentes; además, obtener evidencias sobre los niveles de estigma social hacia la enfermedad mental en función de la edad de los chicos y chicas adolescentes, así como poner de manifiesto su relación con la familiaridad y distintos tipos de conocimientos sobre la enfermedad mental.
Respecto a la estructura interna del $r$ AQ, los resultados no replicaron la estructura multidimensional originalmente propuesta por Watson et al. (2004). El AFE reveló que la versión española del $r$-AQ estaba conformada por una única dimensión en la que se retenían cinco de los ocho ítems que componen la escala original. Esta estructura unidimensional fue confirmada en el AFC, y vino a corroborar la propuesta de Pinto et al. (2012). Una cuestión que se debe destacar sobre los ítems que mostraron un funcionamiento deficiente, es que estaban formulados en sentido inverso. Tradicionalmente, la psicometría recomendaba construir escalas que incluyeran un número equilibrado de ítems positivos (en el sentido del constructo que se va a medir) e inversos (en el sentido opuesto al constructo) para evitar el efecto de aquiescencia o la tendencia a responder, mostrándose de acuerdo con el ítem independientemente de su contenido. Sin embargo, recomendaciones recientes desaconsejan su uso porque pueden generar confusión y dificultades de interpretación, requieren de mayor atención para detectar las partículas de negación (e. g., "Manuel no es peligroso") y pueden alterar la estructura latente de la escala (Van Sonderen, Sanderman, \& Coyne, 2013). De cara a seguir avanzando en esta la cuestión, sería interesante que en futuros estudios se analizara la estructura interna del $\mathrm{r}$-AQ tras transformar los ítems inversos en positivos. De esta manera, se podría determinar con mayor rigurosidad la conveniencia de eliminar o incluir el contenido de estos ítems en la escala final, así como volver a analizar su estructura interna para comprobar la posibilidad de usar una estructura multidimensional. Con esto en mente, lo más recomendable es tomar estos datos con una cierta cautela. De hecho, lo más seguro es concluir que, en líneas generales, los hallazgos de esta investigación se sitúan en consonancia con los de Pinto et al. (2012), mostrando como lo más adecuado el uso de una versión modificada de cinco ítems con una estructura unidimensional.

En relación con las diferencias de género, nuestros resultados se situaron en consonancia 
con la literatura científica previa, pues mostraron que las chicas estigmatizaban menos que los chicos (e. g., Chandra \& Minkovitz, 2006; Holman, 2015; O’Driscoll et al., 2015; Pascucci et al., 2017). No obstante, atendiendo a los tamaños de efecto, las diferencias fueron menos acentuadas de lo que venía encontrándose en el plano internacional (Corrigan et al., 2003). En cuanto a la edad, al contrario de lo que observan los estudios con población adulta (Schomerus et al., 2015), se encontró que los adolescentes de mayor edad presentaban menores niveles de estigma. Estos datos pueden explicarse con base en los estudios sobre desarrollo socioemocional, pues la creciente capacidad para razonar sobre conceptos abstractos y reflexionar desde diferentes perspectivas, unida a la mayor empatía y prosocialidad (Van der Graff, Carlo, Crocetti, Koot, \& Branje, 2018), puede contribuir a que los adolescentes moderen los prejuicios y conductas de discriminación hacia otros compañeros con problemas de salud mental. Pese a ello, los datos de este estudio no permiten descartar que estas diferencias sean debidas al efecto de cohortes generacionales. Es por esto que creemos necesario que investigaciones futuras hagan uso de diseños longitudinales que permitan analizar la trayectoria evolutiva que sigue el estigma social hacia la enfermedad mental.

En cuanto al papel de la familiaridad y el conocimiento sobre la enfermedad mental, de acuerdo con lo esperado, se observó que ambas variables se relacionaban de manera significativa con el estigma social. En la línea de investigaciones previas y estudios de intervención (Chisholm et al., 2016; Simmons, Jones, \& Bradley, 2017), este estudio puso de manifiesto que mayores niveles de familiaridad se relacionaban con menores niveles de estigma. En este sentido, las evidencias procedentes de intervenciones para la reducción del estigma recomiendan que se facilite que chicos y chicas establezcan contacto directo con personas con problemas de salud mental como estrategia para fomentar actitudes más positivas (Griffiths, Carron-Arthur, Parsons, \& Reid, 2014). Asimismo, los datos evidenciaron que los adolescentes que disponían de conocimiento más ajustado sobre la enfermedad mental mostraban menores niveles de estigma. Quizás, una de las cuestiones más interesantes es que de los cinco tipos de conocimiento analizados (prevalencia, síntomas, causas, tratamiento y características) fue el conocimiento sobre las características, evaluado mediante ítems que hacían referencia a mitos y estereotipos frecuentemente mantenidos hacia las personas con enfermedad mental, seguido por el conocimiento sobre la prevalencia, los que mostraron mayor poder predictivo. En cambio, el conocimiento sobre los síntomas y los tratamientos no predecía el estigma social, y el conocimiento relacionado con las causas apenas mostró contribución.

De estos resultados pueden extraerse ciertas implicaciones prácticas para el diseño de intervenciones dirigidas a la reducción del estigma social hacia la enfermedad mental. En primer lugar, es importante tener en cuenta la influencia del género en el estigma. Los datos sugieren que deben reforzarse especialmente los programas dirigidos a chicos. En segundo lugar, la formación combinada ofreciendo a los adolescentes la oportunidad de compartir experiencias directas con personas con problemas de salud mental puede ser una estrategia útil para reducir el estigma. No obstante, es necesario diseñar cuidadosamente los contenidos de la formación. De acuerdo a los datos, los contenidos deberían centrarse en abordar los mitos y estereotipos asociados a las características de las personas con problemas de salud mental y no tanto en proporcionar conocimiento especializado sobre su etiología, síntomas o tratamientos. En este sentido, un análisis exhaustivo de intervenciones psicoeducativas que mostraron escasa efectividad revela que la formación proporcionada versaba sobre las causas neurobiológicas o genéticas de las enfermedades mentales, sus síntomas o los tratamientos (Casados, 2017). En esta línea, algunos estudios de revisión sobre la eficacia de los programas psicoeducativos concluyen que las explicaciones sobre las causas, los síntomas y los tratamientos de las enfermedades mentales pueden contribuir a mejorar su reconocimiento, y fomentar la búsqueda de ayuda, pero no reducen las 
conductas discriminatorias e incluso aumentan las creencias de peligrosidad y culpabilidad (Haslam \& Kvaale, 2015; Schomerus et al., 2012).

Pese a las implicaciones de estos resultados, algunas limitaciones deben ser señalarse. En primer lugar, una de sus principales limitaciones concierne al uso de un diseño de tipo transversal que impidió la interpretación de relaciones entre variables en términos causales, así como el análisis de trayectorias evolutivas. En futuros estudios, sería aconsejable la adopción de un diseño longitudinal que permita examinar la direccionalidad de las relaciones (por ejemplo, si la familiaridad y el nivel de conocimiento predicen cambios en los niveles de estigma), así como examinar la evolución de los niveles de estigma a lo largo de la adolescencia. Igualmente, en cuanto a la forma de evaluación, es necesario dejar constancia de las limitaciones derivadas de la utilización de cuestionarios y escalas autocumplimentadas pues esto puede haber acrecentado la deseabilidad social en las respuestas que concernían a la expresión de actitudes políticamente incorrectas, reduciendo, por tanto, las puntuaciones medias de estigma social hacia la enfermedad mental en chicos y chicas adolescentes. Se considera que futuras investigaciones deberían complementar estas fuentes de información con la observación de interacciones directas con personas con problemas de salud mental, al mismo tiempo que deberían permitir la exploración de los mecanismos que pueden explicar los mayores niveles de estigma encontrados en chicos comparativamente con los de chicas.

Pese a las limitaciones, se considera que los resultados de este estudio contribuyen a mejorar el conocimiento sobre el estigma social hacia la enfermedad mental en la adolescencia, así como sobre los factores que se relacionan con en este. Además, ofrecen información relevante para el diseño de intervenciones para la reducción del rechazo social y la mejora de la integración de las personas que las sufren.

\section{Referencias}

Angermeyer, M. C., Beck, M., \& Matschinger, H. (2003). Determinants of the public's preference for social distance from people with schizophrenia. Canadian Journal of Psychiatry, 48, 663-668. https://doi.org/10.1 177/070674370304801004

Angermeyer, M. C., \& Dietrich, S. (2006). Public beliefs about and attitudes towards people with mental illness: A review of population studies. Acta Psychiatrica Scandinavica, 113, 163-179. https://doi.org/10.1111/j.1600-04 47.2005.00699.x

Casados, A. (2017). Reducing the stigma of mental illness: Current approaches and future directions. Clinical Psychology Science and Practice, 24, 306-323. https://doi.org/10 $.1111 /$ cpsp.12206

Chisholm, K., Patterson, P., Torgerson, C., Turner, E., Jenkinson, D., \& Birchwood, M. (2016). Impact of contact on adolescents' mental health literacy and stigma: The SchoolSpace cluster randomized controlled trial. BMJ Open, 6(2), e009435. https://doi. org/10.1136/bmjopen-2015-009435

Clement, S., Schauman, O., Graham, T., Maggioni, F., Evans-Lacko, S., Bezborodovs, N., \& Thornicroft, G. (2015). What is the impact of mental health-related stigma on help-seeking? A systematic review of quantitative and qualitative studies. Psychological Medicine, 45, 11-27. ht tps://doi.org/10.1017/S0033291714000129

Cohen, J. (1988). Statistical power analysis for the behavioral sciences (2. ${ }^{\mathrm{a}}$ ed.). Hillsdate, NJ: LEA.

Corrigan, P. Q., \& Kosyluk, K. A. (2014). Mental illness stigma: Types, constructs, and vehicles for change. En P. W. Corrigan (Ed.), The stigma of disease and disability: Understanding causes and overcoming injustices (pp. 35-36). Nueva York: American Psychological Association. Corrigan, P. W., Lurie, B., Goldman, H., Slopen, N., Medasani, K., \& Phelan, S. (2005). How adolescents perceive the stigma of mental illness and alcohol abuse. Psychiatric 
Services, 56, 544-550. https://doi.org/10.11 76/appi.ps.56.5.544

Corrigan, P. W., Markowitz, F., Watson, A., Rowan, D., \& Kubiak, M. A. (2003). An attribution model of public discrimination towards persons with mental illness. Journal of Health and Social Behavior, 44, 162-179. h ttps://doi.org/10.2307/1519806

European Comission. (13 de junio de 2008). European Pact for Mental Health and Well-Being. EU high-level conference together for mental health and wellbeing. Bruselas: Autor. Recuperado de http://ec.europa.eu/health/ph_determin ants/life_style/mental/docs/pact_en.pdf

Griffiths, K. M., Carron-Arthur, B., Parsons, A., \& Reid, R. (2014). Effectiveness of programs for reducing the stigma associated with mental disorders. A meta-analysis of randomized, controlled trials. World Psychiatry, 13, 161-175. https://doi.org/10.1 002/wps.20129

Haslam, N., \& Kvaale, E. P. (2015). Biogenetic explanations of mental disorder: The mixed blessings model. Current Directions in Psychological Science, 24, 399-404. https://d oi.org/10.1177/0963721415588082

Hinshaw, S. P. (2006). Stigma and mental illness: Developmental issues and future prospects. En D. Cicchetti (Ed.), Developmental psychopatology (Vol. 3, pp. 841-881). Hoboken, NJ: Wiley.

Holman, D. (2015). Exploring the relationship between social class, mental illness stigma and mental health literacy using British national survey data. Health, 19, 413-419. h ttps://doi.org/10.1177/1363459314554316

Horn, J. L. (1965). A rationale and test for the number of factors in a factor analysis. Psychometrika, 30, 179-185. https://doi.org/ 10.1007/BF02289447

Kline, R. (2011). Principles and practice of structural equation modeling. Nueva York: Guilford.

Lloret, S., Ferreres, A., Hernández, A., \& Tomás, I. (2014). El análisis factorial exploratorio de los ítems: una guía práctica, revisada y actualizada. Anales de Psicología, 30,
1151-1169. https://doi.org/10.6018/analesp s.30.3.199361

Martínez, A. G., \& Hinshaw, S. P. (2016). Mental health stigma: Theory, developmental issues, and research priorities. En D. Cicchetti (Ed.), Developmental psychopathology (3.a ed., pp. 997-1039). New Jersey, NJ: Wiley.

Mukolo, A., Heflinger, C. A., \& Wallston, K. (2010). The stigma of childhood mental disorders: A conceptual framework. Journal of the American Academy of Child and Adolescent Psychiatry, 49, 92-198. https://do i.org/10.1016/j.ypsy.2010.10.021

Muñoz, M., Guillén, A. I., Pérez-Santos, E., \& Corrigan, P. (2015). A structural equation modeling study of the Spanish Mental Illness Stigma Attribution Questionnaire (AQ-27-E). American Journal of Orthopsychiatry, 85, 243-249. https://doi.org/10.1037/ort00000 59

Muthén, L. K., \& Muthén, B. O. (2012). Mplus User's Guide Statistical Analysis with Latent Variables (7.a ed.). Los Angeles: Muthén \& Muthén.

National Academies of Sciences, Engineering, and Medicine (2017). Approaches to reducing stigma. National Academy of Sciences. In Ending discrimination against people with mental and substance use disorders: The evidence for stigma change (pp. 69-92). National Academies Press.

Navas, M. J., Fidalgo, A. M., Gabriel, C., Suárez, J. C., Brioso, A., Gil, G., ... Sarriá, E. (2001). Métodos, diseños y técnicas de investigación psicológica. Madrid: Universidad Nacional de Educación a Distancia.

O'Driscoll, C., Heary, C., Hennessy, E., \& McKeague, L. (2015). Adolescents' explanations for the exclusion of peers with mental health problems: An insight into stigma. Journal of Adolescent Research, 30, 710-728. https://doi.org/10.1177/07435584 14550246

Organización Mundial de la Salud. (2018). Salud mental del Adolescente. 
Datos y cifras. Recuperado de https://www.who.int/es/news-room/facts heets/detail/adolescent-mental-health

Pascucci, M., Montagna, M., Di Sabatino, D., Stella, E., Nicastro, R., Grandinetti, P., ... \& Bellomo, A. (2017). Stigma and attitudes towards mental illness: Gender differences in a sample of Italian medical students. European Psyciatry, 41, S739. https://doi.org /10.1016/j.eurpsy.2017.01.1359

Petersen, K., Soegaard, V., Lodahl, B., Vinther, C., \& Wind, G. (2014). Recovery from mental illness: A service user perspective on facilitators and barriers. Community Mental Health Journal, 51, 1-13. https://doi.org/10. 1007/s10597-014-9779-7

Pettigrew, T. F., \& Tropp, L. R. (2008). How does intergroup contact reduce prejudice? Metaanalytic tests of three mediators. European Journal of Social Psychology, 38, 922-934. ht tps://doi.org/10.1002/ejsp.504

Pinto, M., Hickman, R., Logsdon, C., \& Burant, C. (2012). Psychometric evaluation of the revised-Attribution Questionnaire ( $\mathrm{r}-\mathrm{AQ})$ to measure mental illness stigma in adolescents. Journal of Nursing Measurement, 20, 47-58. https://doi.org/10. 1891/1061-3749.20.1.47

Quinn, D. M., Williams, M. K., \& Weisz, B. M. (2015). From discrimination to internalized mental illness stigma: The mediating roles of anticipated discrimination and anticipated stigma. Psychiatric Rehabilitation Journal, 38, 103-108. https://doi.org/10.103 $7 /$ prj0000136

Reavley, N., \& Jorm, A. (2011). Stigmatizing attitudes towards people with mental disorders: findings from an Australian national survey of mental health literacy and stigma. Australian Eु New Zealand Journal of Psychiatry, 45, 1086-1093. https:/ /doi.org/10.3109/00048674.2011.621061

Schnittker, J. (2000). Gender and reactions to psychological problems: An examination of social tolerance and perceived dangerousness. Journal of Health and Social Behaviour, 41 (2), 224-240. https://doi.org/1 $0.2307 / 2676307$
Schomerus, G., Schwahn, C., Holzinger, A., Corrigan, P. W., Grabe, H. J., Carta, M. G., \& Angermeyer, M. C. (2012). Evolution of public attitudes about mental illness: A systematic review and metaanalysis. Acta Psychiatrica Scandinavica, 125, 440-452. https://doi.org/10.1111/j.160 0-0447.2012.01826.x

Schomerus, G., Van der Auwera, S., Matschinger, H., Baumeister, S. E., \& Angermeyer, M. C. (2015). Do attitudes towards persons with mental illness worsen during the course of life? An age-period-cohort analysis. Acta Psychiatrica Scandinavica, 132, 357-364. htt ps://doi.org/10.1111/acps.12401

Seacat, J. (2014). Mental health stigma update: A review of consequences. Advances in Mental Health, 12, 202-215. https://doi.org/10.108 0/18374905.2014.11081898

Simmons, L., Jones, T., \& Bradley, E. (2017). Reducing mental health stigma. The relationship between knowledge and attitude change. European Journal of Mental Health, 12, 25-40. https://doi.org/10.5708/E JMH.12.2017.1.2

Van der Graff, J., Carlo, G., Crocetti, E., Koot, H. M., \& Branje, S. (2018). Prosocial behavior in adolescence: Gender differences in development and links with empathy. Journal of Youth and Adolescence,47, 1086-1099. https://doi.org/ 10.1007/s10964-017-0786-1

van Sonderen, E., Sanderman, R., \& Coyne, J. C. (2013). Ineffectiveness of reverse wording of questionnaire items: Let's learn from cows in the rain. PLOS One, 8(7), e68967. https://doi.org/10.1371/journal.po ne.0068967

Vigo, D., Thornicroft, G., \& Atun, R. (2016). Estimating the true global burden of mental illness. The Lancet Psychiatry, 3, 171-178. https://doi.org/10.1016/S2215-03 66(15)00505-2

Wahl, O. (2002). Children's views of mental illness: A review of the literature. Psychiatric Rehabilitation Skills, 6, 134-158. https://doi. org/10.1080/10973430208408430 
Wainberg, M. L., Scorza, P., Shultz, J., Helpman, L., Mootz, J. J., Johnson, K. A., ... Arbuckle, M. (2017). Challenges and opportunities in global mental health: A research-to-practice perspective. Current Psychiatry Reports, 19(5), 28. https://doi.org /10.1007/s11920-017-0780-z

Watson, A., Otey, E., Westbrook, A., Qander, A., Lamb, T., Corrigan, P. W., \& Fenton, W. (2004). Changing middle schoolers' attitudes about mental illness through education. Schizophrenia Bulletin. The Journal of Psychoses and Related Disorders, 30(3), 563-572. https://doi.org/10.1093/oxf ordjournals.schbul.a007100

Wei, Y., McGrath, P., Hayden, J., \& Kutcher, S. (2018). The quality of mental health literacy measurement tools evaluating the stigma of mental illness: A systematic review. Epidemiology and Psychiatric Sciences, 27, 433-462. https://doi.org/10.1017/S2045 796017000178

Weiner, B. (1995). Judgementsof responsibility: A foundation for a theory of social conduct. Nueva York: Guilford.

\section{Notas}

* Artículo de investigación.

1 El análisis paralelo fue desarrollado utilizando un estimador robusto de máxima verosimilitud (MLR) ya que el software de análisis utilizado no permite implementarlo con matrices de correlaciones policóricas. 